\title{
Systematic and integrative reviews: synthesising evidence for community nursing practice.
}

\author{
KENNEDY, C., RAMUKUMBA, M.M.
}


Title: Systematic and integrative reviews; synthesising evidence for community nursing practice

\section{Authors}

Catriona Kennedy

QNIS Professor of Community Nursing

School of Nursing and Midwifery

Robert Gordon University

Aberdeen

AB10 7QG

c.m.kennedy1@rgu.ac.uk

Mokholelana Margaret Ramukumba

Professor: Department of Health Studies

University of South Africa

Muckleneuk

Pretoria

ramukmm@unisa.ac.za

Conflict of interest: The authors confirm they have no conflict of interest in respect of this paper. 


\section{Abstract}

Ease of access to vast amounts of information presents significant opportunities and challenges for nurses in the community as they seek to base their practice on the 'best' available evidence. Growing expectations around evidence based practice have developed alongside developments in evidence synthesis which adopts robust approaches to identifying, appraising and synthesising key evidence for clinical decision making. The context in which evidence based practice occurs is key and we discuss here the skills and knowledge needed for community nurses to discern how evidence and information should influence their decisions to review and change approaches to clinical practice. Importantly, if nurses understand the status of evidence underpinning areas of practice they can ensure the preferences and needs of patients and families are met.

\section{Key words}

Evidence based practice, evidence synthesis, clinical decision making 


\section{Key points}

- The exponential growth in knowledge and ease of access to vast amounts of information presents significant opportunities and challenges for nurses in the community as they seek to implement evidence based practice.

- Evidence synthesis, the process of analysing the findings from all the studies on a topic together in context using a robust and transparent method, informs EBP and is now a global endeavour.

- EBP is complex and in order to base clinical decisions and actions on the best available evidence community nurses needs to understand evidence and approaches to the synthesis of evidence.

- If nurses understand the status of evidence underpinning areas of practice they can ensure the preferences and needs of patients and families are met.

\section{Key points}

- What areas of your practice do you think are amenable to EBP?

- Where is that evidence drawn from?

- How confident are you that the evidence is robust enough to justify developing or changing something you do?

- How do you take in to account the needs and preferences of patients and families? 


\section{Introduction and background}

The exponential growth in knowledge and ease of access to vast amounts of information presents significant opportunities and challenges for nurses in the community. Expectations that nurses will base their practice on the 'best' available evidence have developed alongside the availability of online resources, graduate preparation of nurses and consumer expectations. Nurses in the community require knowledge and skills to discern how evidence and information should influence their decisions to review and change approaches to clinical practice.

In 1983 Dr Lisbeth Hockey said 'Nurses who really care, in the true sense of the term, will not be content. They will question, read and avail themselves of the new knowledge for the benefit of their patients and clients'. Few could have predicted the growth in knowledge and its availability since the early 1980s. Critical to this are the skills of nurses in accessing, appraising and evaluating the quality of information to inform changes to practice.

The aim of this paper is to inform professional dialogue and to appraise the expectations around evidence-based practice (EBP) for community nursing practice. Developments in EBP and approaches to evidence synthesis are presented. This includes accessing, appraising, analysing and synthesising evidence and judging clinical relevance drawing on practice examples.

This paper is timely given BJCN and a range of other nursing journals commission and publish evidence summaries (eg Barrett 2019, Kennedy 2019) through the Cochrane Nursing Care Field. Evidence summaries provide clinicians with a synthesised synopsis of key evidence from the academic literature of a clinical issue/question/problem. Importantly, they identify the implications for nursing practice and through the rigorous assessment of the quality of available evidence, provide guidance for EBP and clinical decision-making. 


\section{Evidence based practice}

Despite the well documented need for, and benefits of EBP, literature reveals various barriers and challenges to its successful implementation. These include lack of time, workload, knowledge and skills about research and evidence and lack of nursing autonomy (Dalheim et al. 2012 Grant et al. 2012). Community nurses face a range of complex situations in the households, communities and the range of diverse stakeholders they work with across health and social care and the third sector. It is therefore important they have time and the skills to identify clinical issues/problems, reflect on current clinical practice and incorporate researchbased knowledge into their clinical practice. However, in many contexts this may require an ideological shift, supportive leadership, clients who are aware of their role in healthcare decisions and organisational culture (Edwards et al. 2002)

In a recent qualitative study, Teodorowoski et al (2019) explored community nurses experiences of EBP. Three pathways to change were proposed by participants as reflecting their experiences: bottom-up, top-down and collaborative pathways. There pathways are not mutually exclusive and there exists overlap but the nature of the proposed change, the available evidence, 'buy in' from colleagues and issues around implementation are key to the approach taken. These findings identify a need for practitioners to be supported through a complex process of change with opportunities for ongoing education, robust managerial support, accessible online resources and support through a practice development role. Forming collaborative partnerships between academia and practice could be one of the options to strengthen EBP.

The context in which community nurses work is important and those in resource-constrained countries are confronted with a myriad of complex situations. The changing patterns of disease, re-emergence of previously controlled infectious diseases and antibiotic resistance, are key challenges. This constant change creates opportunities for community health practitioners to initiate the process of evidence synthesis and the creation of new and resourceful ways of providing healthcare in the community. 
The context driven nature of EBP, alongside the need to recognize the autonomy and beliefs systems of patients and families, signify the complexity involved in identifying a need for change and the need for community nurses to formulate critical questions about their practice. Therefore, advocacy for enhanced collaborations between knowledge producers (researchers) and knowledge translators (practitioners) might address the need to translate research results into practice (Banner et al 2019).

Policy is influential to EBP and can be the main driver for change, often reflecting a 'top down' approach (Teodorowski et al 2019). Davies (2014) defines knowledge translation as the process of mobilising evidence into heath policy and service delivery and this is the essence of EBP for many areas of health care practice. Stakeholder agreement and the co-creation of new possibilities lie at the heart of EBP alongside opportunities to enact new theories of care, increase client satisfaction with care and ensure the competence of practitioners.

Implementing EBP into routine clinical practice, even when there is a strong and convincing case to do so, is complex and requires to be planned. There is an increasing literature in implentation science which community nurses might usefully access (e.g. Bauer, Damschroder, Hagedorn et al 2015). In order to base clinical decisions and actions on the best available evidence community nurses need to understand evidence and approaches to the synthesis of evidence.

\section{Evidence synthesis.}

Parallel to developments in EBP, approaches to evidence synthesis are now a global endeavour. For the purposes of this paper, we are defining evidence synthesis as the interpretation of individual studies within the context of global knowledge for a given topic. These syntheses provide a rigorous and transparent knowledge base for translating research into decisions. The advantage of evidence synthesis is that all studies on a topic are assessed together in context' (evidencesynthesis.org accessed 26/02/19). 
Evidence synthesis, the process of analysing the findings from all the studies on a topic together in context using a robust and transparent method, underpins EBP. Evidence synthesis provides information, which pools available evidence through a transparent method, rather than individual studies that may or may not be free from bias. Therefore such synthesis have an important role to play in EBP

Established in 1993, the Cochrane collaboration was developed primarily in response to calls for evidence-based medicine (Sackett et al 1996). Cochrane is an independent and global endeavour with contributors from across 130 countries to 53 topic groups. The main output from Cochrane is systematic reviews which follow a rigorous methodology and peer review process at each stage of title registration, protocol development and completion of the review. REVMAN software is used to summarise the best available evidence from primary empirical studies and completed reviews are published in the Cochrane library. Cochrane reviews are arguably the 'gold standard' in evidence synthesis given the rigour with which they are developed and the authors' responsibility to update reviews at regular intervals (normally 2-4 years) to ensure new knowledge is captured. Establishing the clinical relevance of review findings, public engagement, dissemination, developing methods of research synthesis and appraising the contextual issues around implementation of research findings into clinical practice are also priority areas for the collaboration (https://uk.cochrane.org/about-us Accessed 31/10/19).

Cochrane mainly focusses on evidence from randomised controlled trials and other quantitative approaches involving an intervention and comparator group. There are developments in qualitative synthesis in Cochrane but to date they are recognised as the main producers of quantitative systematic reviews. Cochrane reviews have been criticised as not relevant to all questions and interventions of interest to nursing. As in primary research methods 'one size' does not fit all, and nor should they given the complex and transactional nature of nursing practice. Evidence synthesis as a methodology has a range of approaches and tools and it is beyond the scope of this paper to present all of these. 
The Joanna Briggs Institute (JBI) was established in 1996 and as part of the evidence based movement its focus has been on establishing collaborating centres and training systematic reviewers. The main outputs from JBI are 'Best Practice Information Sheets' to inform clinical decision making at the point of care delivery. JBI best practice statements draw on a wide range of available evidence to inform clinical questions (https://joannabriggs.org Accessed 07/11/19). It is important to note that Cochrane reviews focus mainly on Randomised Controlled trials, which are considered to be the most robust research design. $\mathrm{JBI}$ reviews and the integrative review approach detailed below, are more inclusive. When reviewing the findings of Cochrane and other types of review it is important to understand the 'confidence' you can attribute to them.

It is not a requirement to align to an organisation such as Cochrane or JBI and many systematic and integrative reviews are published which report a clear methodology. Another approach which is appropriate to clinical questions in the 'integrative review methods proposed by Whittemore and Knafl (2005). The purpose of integrative reviews is to synthesise all the available evidence be it quantitative or qualitative and conduct a descriptive thematic presentation of results.

Regardless, there are some common features of the systematic approach to evidence synthesis and these are detailed in Table 1. The differences in how these are applied lie in the nature of the review questions, available evidence, approaches to analysis and contextual relevance. Quality appraisal is relevant to Cochrane and integrative reviews and sets this approach to reviewing the literature apart from narrative reviews. Given the need for the judicious use of evidence there is arguably no longer a place in health care practice and decision making for the narrative approach.

Insert Table 1

Having established the links between EBP and evidence synthesis what does this mean for nurses in the community and their day-to-day work? Nurses in the community need to make 
decisions about their work with people, patients, families and communities. Given the complex nature of EBP and discerning the quality of evidence available to inform decision making we now present a clinical scenario which illuminates some of the challenges of combining policy, practice and evidence drawing on an example relevant to clinical practice - metastatic spinal cord compression'

\section{The case of metastatic spinal cord compression}

Metastatic spinal cord compression (MSCC) is a significant complication of advanced, progressive cancer and an oncological emergency. An acute, complex onset condition the secondary spread of cancer to the spinal cord can result in paraplegia within 24 hours if not diagnosed and treated quickly. People presenting with MSCC are likely to have short life expectancy and a paradox may exist for clinicians in managing an acute onset medical emergency and a patient in need of palliative and end of life care. The palliative care approach identifies patient preferences, quality of life and physical, social and emotional support as key goals. Once a confirmed diagnosis of MSCC is made nurses caring for people with MSCC lack clear evidence to inform patient positioning (mobliisation) and bracing for pain relief and spinal stability in adults (Lee et al 2015).

NICE guidelines (2008) exist and these cover a range of aspects linked to MSCC from initial diagnosis and some advice about positioning, mobilisation and rehabilitation for people with MSCC (https://www.nice.org.uk/guidance/CG75/chapter/1-Guidance\#supportive-care-andrehabilitation Accessed 31/07/19). The NICE guidelines were reviewed in February 2019 but no new evidence was uncovered on patient positioning and bracing for pain relief and spinal stability (https://www.nice.org.uk/guidance/cg75/resources/2019-surveillance-of-metastaticspinal-cord-compression-in-adults-risk-assessment-diagnosis-and-management-niceguideline-cg75-6665002669/chapter/Overview-of-2019-surveillancemethods?tab=evidence\#evidence-considered-in-surveillance accessed 31/07/19). Lee et als (2012 and updated 2015) Cochrane systematic did not identify any RCTs about patient positioning and bracing for pain relief and spinal stability in adults. Given the absence of 
RCTs the authors concluded a lack of evidence based guidance around how to correctly position and when to mobilise patients with MSCC or whether spinal bracing is an effective technique for reducing pain or improving quality of life. In the absence of robust evidence clinical decision making about mobilising patients, bed rest and keeping the person flat are subject to individual clinician preferences and may not reflect patient preferences and quality of life aspects of palliative, end of life care.

Kilbride et al (2010) conducted an integrative review of 35 papers to synthesise the available evidence about the management of MSCC. They concluded the evidence related to spinal stability, bracing, patient mobilisation and positioning is limited and inconclusive. Patients with MSCC and a poor prognosis should therefore have individual preferences and quality of life considered. These findings are corroborated by Lawton et al (2018) who recommend consideration of the patient's goals and psychosocial needs when agreeing a treatment plan for MSCC.

Given the evidence around the best approaches to managing aspects of MSCC is limited and inconclusive how do practitioners decide what to do? Patients and families need care and advice when coping with the challenges of MSCC so the needs and preferences of the patient should be central to joint decision making. In this case there exists no clear evidence to guide the actions of the community nurse. It is therefore important that practitioners know and understand there is not conclusive evidence to support interventions such as nursing the patient flat or immobilising them. This allows the nurse to focus on the needs and preferences of the patient rather than implementing a routine that lacks rigorous evidence to underpin it.

\section{Discussion}

EBP and evidence synthesis are important concepts for community nurses to understand and embrace. We have discussed the relationship between these areas and identified the complexity of clinical decision making when considering the available evidence, the quality 
appraisal of that evidence and whether a particular intervention or approach is justified and the needs and preferences of patients and families. Recognising different pathways to the implementation of EBP can help community nurses determine what is driving the change and help them to better discern the appropriate responses to the clinical, policy or patient specific issue or problem (Teodorowski et al 2019).

The contextual and relational aspects of clinical practice need to be recognised in EBP and whilst community nurses need to be able to source and understand evidence, patient preferences and needs must be considered. In many respects, current agendas around EBP, evidence synthesis and co-production and creation of care decisions are at odds with each other. Arguably EBP suggests there exists a 'right' way to do things drawing on evidence synthesis to inform actions. The context and everyday realities of working together with people demonstrate this is far from straightforward.

\section{Conclusion}

We have identified a number of challenges around the intersection of practice, policy and evidence. No more so than in the present climate community nurses need to embrace and engage in the developments around evidence synthesis to ensure delivery of the best care based on the best available evidence if it exists. Importantly, if nurses understand the status of evidence underpinning areas of practice they can ensure the preferences and needs of patients and families are met. 


\section{References}

Banner D, Bains M, Carroll S et al. Patient and Public Engagement in Integrated Knowledge Translation Research: Are we there yet? BMC. 2019. 5:8. https://doi.org/10.1186/s40900$\underline{019-0139-1}$

Barrett D. Admission avoidance: hospital at home Br J Community Nurs. May 2019 24,5

Bauer M, Damschroder L, Hagedorn H et al. BMC Psychology. (2015) 3:32

Brown CE, Wickline MA, Glaser D. Nursing practice, knowledge, attitudes and perceived barriers to evidence-based practice at an academic medical center. J Adv Nurs. 2009 65(2):371-81. doi: 10.1111/j.1365-2648.2008.04878.

Cochrane UK About us https://uk.cochrane.org/about-us accessed 31/10/19

Dalheim A, Harthug S, Nilsen RM et al. Factors influencing the development of evidencebased practice among nurses: a self-report survey. BMC Health Serv Res. 2012 12, 367 doi:10.1186/1472-6963-12-367

Davies J. Nursing \& Health Survival Guide: Evidence Based Practice. 2014. Routledge Tailor \& Francis Group NY USA.

Edwards $\mathrm{H}$, Chapman $\mathrm{H}$ \& Davis L. Utilization of research evidence by nurses. Nurs and Health Sci. 2002 4(3), pp. 89-95.

Evidence Synthesis International. What is evidence synthesis? https://evidencesynthesis.org/what-is-evidence-synthesis/ accessed 31/10/19

Grant H, Stuhlmacher A \& Bonte-Eley S. Overcoming barriers for research utilization and evidence based practice among staff nurses. J Nurses Staff Dev. 2012. 28 (4) pp. 163-165

Joanna Briggs Institute: Home. https://joannabriggs.org Accessed 07/11/19

Kennedy C. Compulsory Community and Involuntary Outpatient Treatment for People with Severe Mental Disorders. Issues Ment Health Nurs. 2019. 40:6, 537-538, DOI: 10.1080/01612840.2019.1600359

Kilbride L, Cox M, Kennedy C, Lee SH \& R Grant. Metastatic Spinal Cord Compression: A Review of Practice and Care. J Clin Nur. 2010. 19, 1767-1783 doi: 10.1111/j.13652702.2010.03236.x

Lawton A, Lee K, Cheville A, Ferrone M, Rades D \& T Balboni. Assessment and management of patients with metastatic spinal cord compression: a multidisciplinary review J Clin Oncol. 2019 Jan 1;37(1):61-71. doi: 10.1200/JCO.2018.78.1211.

Lee SH, Grant R, Kennedy C, Kilbride L. Positioning and spinal bracing for pain relief in metastatic spinal cord compression in adults. Cochrane Database of Systematic Reviews 2015, Issue 9. Art. No.: CD007609. DOI: 10.1002/14651858.CD007609.pub3.

NICE. Metastatic spinal cord compression in adults: risk assessment, diagnosis and management. 2008 https://www.nice.org.uk/guidance/CG75/chapter/1Guidance\#supportive-care-and-rehabilitation Accessed 31/10/19

Sackett DL, Rosenberg WMC, Gray JAM et al. Evidence based medicine: What it is and what it isn't. BMJ. 1996. 312(7023):71. 
Teodorowski P, Cable C, Kilburn S \& C Kennedy Enacting evidence-based practice: pathways for community nurses. Br J Community Nurs. 2019.24;8 370-376

Whittemore R \& Knafl K. The integrative review: Update methodology J Adv Nurs. 2005 52(5):546-553 


\begin{tabular}{|ll|}
\hline & Table 1: Attributes of systematic and integrative reviews \\
\hline$\bullet$ & Formulation of a review/research question \\
\hline$\bullet$ & Development of a robust search strategy to include key words, databases, search \\
& limits and results \\
\hline$\bullet$ & Data evaluation and analysis - exclusion and inclusion criteria \\
\hline$\bullet$ & Presentation/synthesis of results \\
\hline$\bullet$ & Researcher makes decisions and choices along the way - possibility of error \\
\hline$\bullet$ & Explicit inclusion/exclusion criteria \\
\hline$\bullet$ & Selection and analysis of data by two people \\
\hline$\bullet$ & Presentation of results in tables \\
\hline$\bullet$ & Reasons for exclusion documented \\
\hline$\bullet$ & Assessment of strength of evidence \\
\hline
\end{tabular}

